\title{
A widely adaptable approach to generate integration-free iPSCs from non-invasively acquired human somatic cells
}

\section{Dear Editor,}

Human pluripotent stem cells including human embryonic stem cells (ESCs) and induced pluripotent stem cells (iPSCs) are cells displaying abilities of unlimited self-renewal and differentiation into any somatic cell type. These unique properties make them increasingly attractive for novel applications in disease modeling, drug discovery, and cell therapy (Buganim et al., 2014; Liu et al., 2011; Liu et al., 2012; Sanchez Alvarado and Yamanaka, 2014). Moreover, iPSCs hold great potential for personalized cell therapy as they avoid some of the ethical concerns as well as the immunological rejection issues ascribed to ESCs. To date, iPSCs can be generated from various cell sources that range from commonly used skin fibroblasts to rarely employed blood cells, mostly through virus-based reprogramming methods with different efficiencies (Patel and Yang, 2010). However, the isolation of these original human cells often involves an invasive sample acquisition procedure. Therefore, human somatic cells that can be conveniently obtained in a non-invasive manner, including human endometrium cells (EMCs) and human urine-derived cells (UCs), may represent more convenient and promising sources for the generation of iPSCs. This is especially crucial for patients hypersensitive to any invasive operation (i.e. patients with Diabetes mellitus (DM) and patients with hemophilia). On the other hand, non-integrative reprogramming methods including those mediated by episomal vectors (Okita et al., 2011), Sendai virus vectors (Seki et al., 2012) and small chemicals (Hou et al., 2013; Lin et al., 2009), are increasingly regarded as superior alternatives to viral approaches. Recently, an elegant study has demonstrated that iPSCs could be established by an episomal system comprised of miR-302-367 from human UCs (Xue et al., 2013). Here we report on the generation of integration-free human endometrium-derived iPSCs (emiPSCs) and urine-derived iPSCs (uiPSCs) by a modified episomal reprogramming system. Subsequently, we show that these iPSCs can robustly differentiate into several valuable lineagespecific cell types, including pancreatic progenitor cells (PPs) and neural stem cells (NSCs).

Healthy and patient donors at an age ranging from 8 to 83 were recruited with signed and approved consents by our institutional committees. 1-3 mL menstrual blood or 200-500 $\mathrm{mL}$ urine was centrifuged and washed by phosphate-buffered saline (PBS) containing Penicillin-Streptomycin, and then cultured in mesenchymal cell medium and renal epithelial cell medium respectively for 1-2 weeks. In total, we established 5 EMC lines from healthy donors and $16 \mathrm{UC}$ lines from healthy individuals and type II DM patients. These primary EMCs and UCs proliferate well and could be passaged for at least 6 passages in culture. Next, two EMC lines and two UC lines from healthy individuals and one UC line from a DM patient were randomly chosen and subjected to cellular reprogramming. A normal fibroblast line isolated from a 9-year old donor (Liu et al., 2011) was used as the reprogramming control. Here, we utilized a modified system by which integration-free iPSCs have been successfully generated from fibroblasts of a FANCA mutant Faconi anemia (FA) patient (Liu et al., 2014; Seki et al., 2012). This approach was proven to be robust as FA patient fibroblasts were reported to be refractory to somatic cell reprogramming (Liu et al., 2014; Raya et al., 2009). We also proved that cells reprogrammed through this strategy maintained genomic integrity even in a compromised disease context, as FA patient cells have a defective DNA repair system (Liu et al., 2014; Raya et al., 2009). To initiate cell reprogramming, we electroporated a cocktail of episomal vectors that encode for human OCT4, SOX2, KLF4, LIN28, L-MYC, and a p53specific shRNA, into EMCs and UCs respectively. Sodium butyrate was included in the medium to facilitate cell reprogramming-associated epigenetic remodeling (Liu et al., 2014; Mali et al., 2010). After seeding on top of mouse embryonic fibroblast (MEF) feeders, small iPSC-like colonies appeared around 10 days after electroporation for all selected lines. Typical iPSC colonies with human ESC morphology were ready for picking and expanding at around day 20 (Fig. 1A). All resulting iPSC lines demonstrated typical ESC features, including a high nucleus/cytoplasma ratio, positive alkaline phosphatase (AP) activity, expression of pluripotency markers OCT4, SOX2, and NANOG, as well as demethylation of the OCT4 promoter (Fig. 1A-D). These cells were stably cultured on MEF or Matrigel for more than 20 passages, without acquiring any karyotypic aberrance 

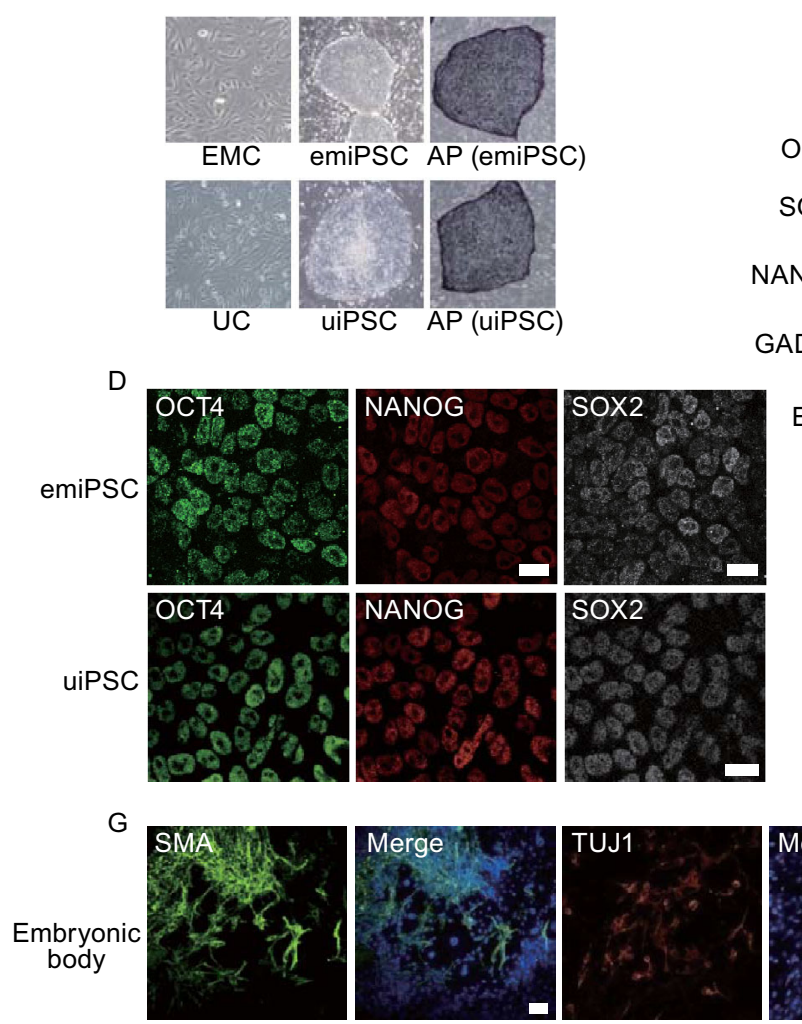

$\mathrm{H}$
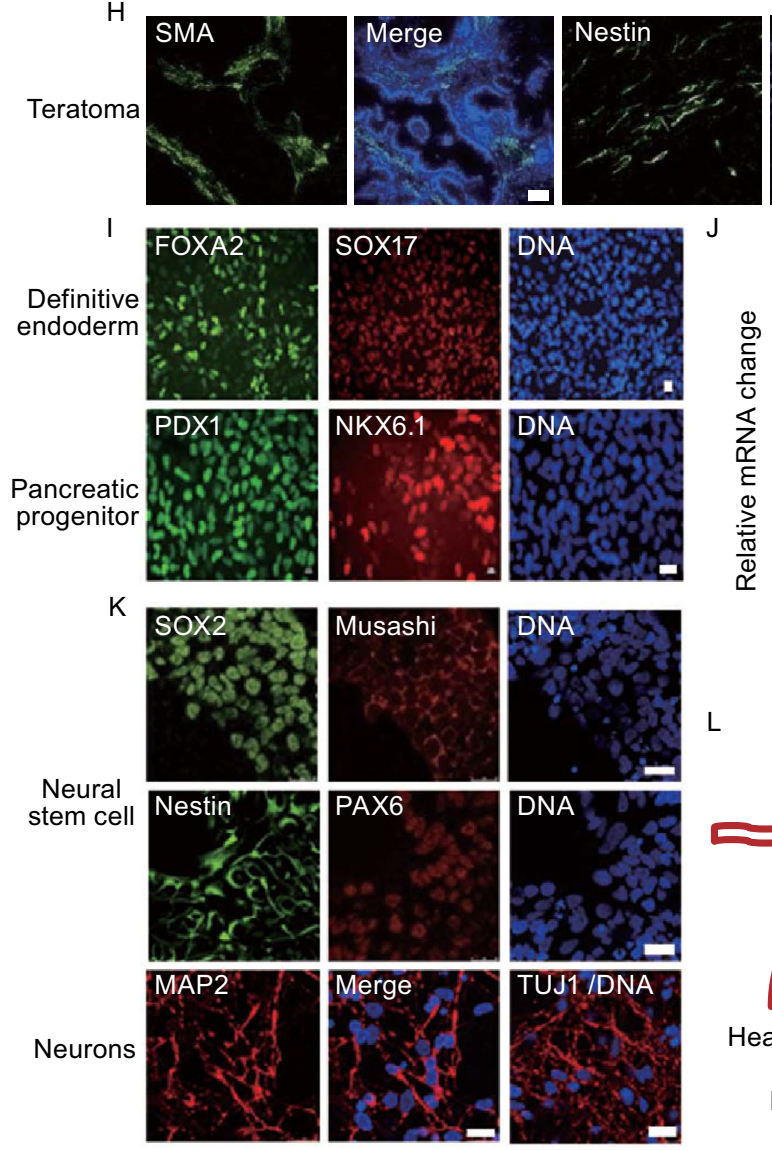

$\mathrm{L}$
B

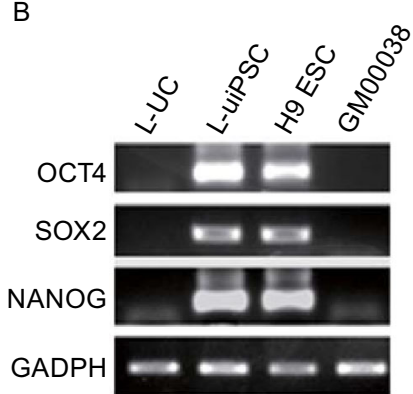

C
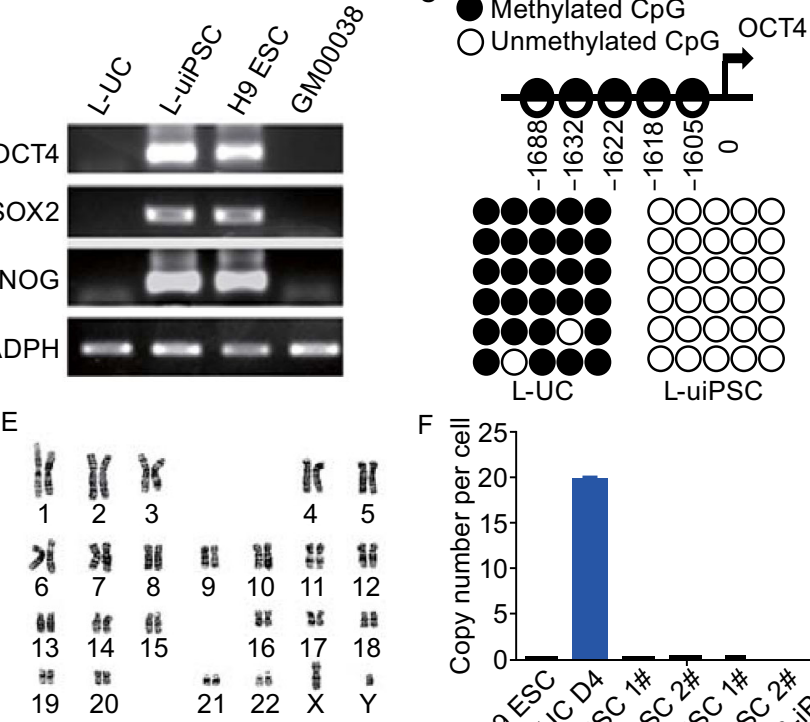

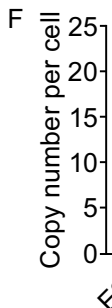
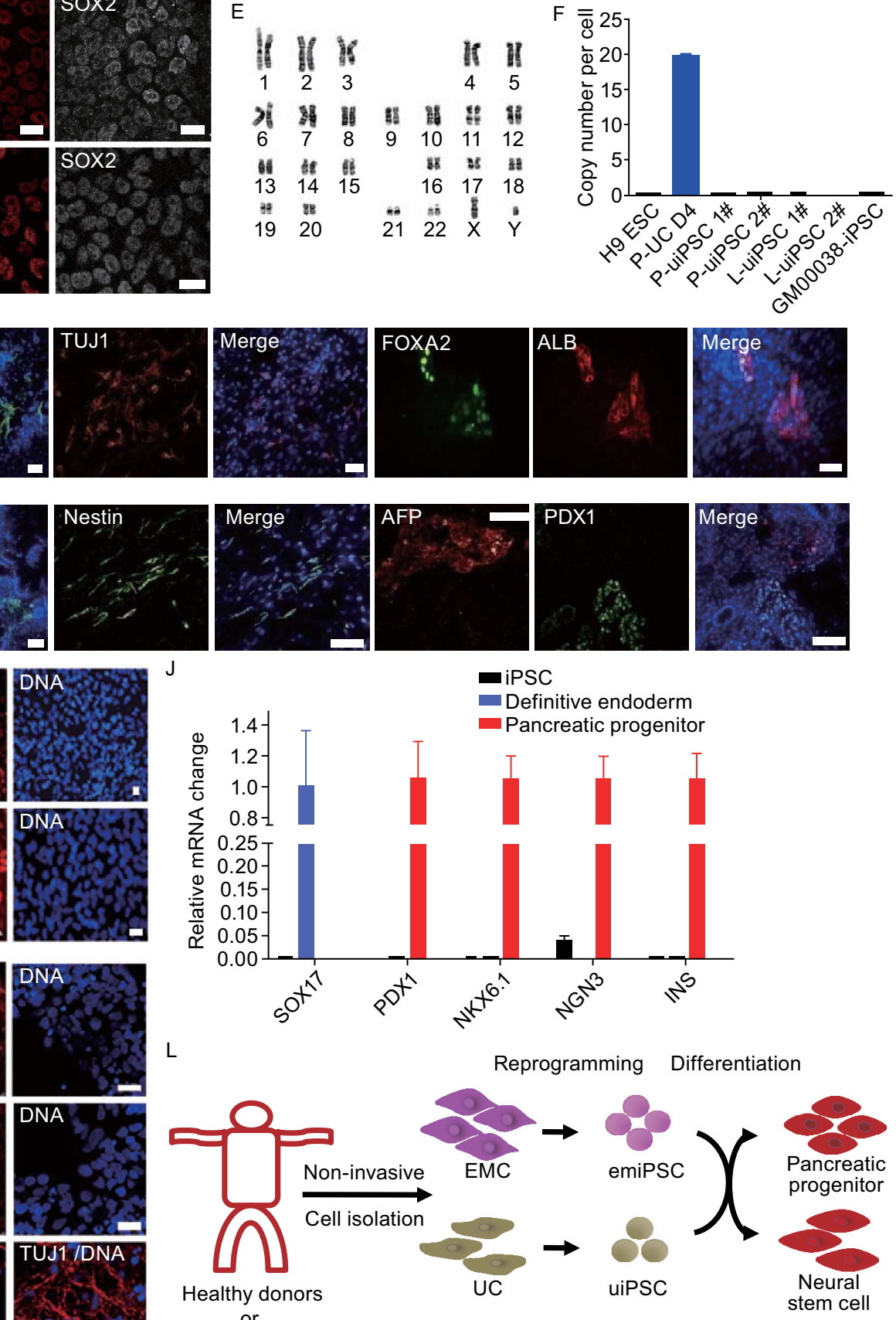
Figure 1. Establishment and characterization of emiPSCs and uiPSCs. (A) Cellular morphology and AP staining of emiPSCs and uiPSCs generated from human EMCs and UCs. (B) RT-qPCR analysis of endogenous pluripotency gene expression in indicated lines. H9 human ESCs and GM00038 human fibroblasts were used as positive and negative controls, respectively. (C) DNA methylation analysis of the OCT4 promoter in the L-UC and L-uiPSC lines. The positions of the CpG dinucleotides relevant with the OCT4 transcription start site are present. (D) Immunofluorescence analysis of pluripotency markers OCT4, NANOG, and SOX2 in L-uiPSCs. Nuclear DNA was stained with Hoechst 33258. (E) G-band analysis of L-uiPSCs showing a normal karyotype. (F) Copy number quantification of the episomal vector element EBNA1 in indicated iPSC lines. H9 human ESCs were employed as a negative control, and the P-UCs 4 days (P-UC D4) after electroporation with the episomal reprograming factors were used as a positive control. (G-H) Spontaneous EB differentiation in vitro $(\mathrm{G})$ and teratoma analysis in vivo $(\mathrm{H})$ verify the differentiation potentials of iPSCs towards endodermal, mesodermal, and ectodermal tissues. Scale bar, $75 \mu \mathrm{m}$ for the teratoma analysis. (I-J) Immunofluorescence analysis (I) and RT-qPCR (J) demonstrate the up-regulation of FOXA2, SOX17 in DE; and up-regulation of PDX1, NKX6.1, NGN3, and INS in PPs. (K) Immunofluorescence analysis shows NSCs generated from iPSCs express specific makers SOX2, Musashi, Nestin, and PAX6; and neurons generated from iPSCs express neuronal makers MAP2 and TUJ1. All scale bars represent $25 \mu \mathrm{m}$ unless otherwise specified. (L) Schematic presentation of establishing integration-free emiPSCs and uiPSCs and generating their PP and NSC derivatives in vitro.

(Fig. 1E). Additionally, quantitative RT-PCR (RT-qPCR) analysis showed no residual episomal vector element in the established iPSC lines (Fig. 1F).

We further examined the in vitro and in vivo differentiation potentials of the generated iPSCs. The in vitro spontaneous differentiation potential of iPSCs was investigated by embryoid body (EB)-based differentiation assays. As shown in Fig. 1G, iPSCs effectively differentiated into TUJ1 (ectoderm), SMA (mesoderm), as well as FOXA2 and ALB (endoderm)-positive cells upon spontaneous differentiation. Furthermore, after injection into immune-deficient NOD/ SCID mice, iPSCs were able to develop into teratomas composed of cells from all three germ-layers marked by SMA, AFP, PDX1, and Nestin expressions (Fig. 1H).

Next, we investigated the potency of the iPSCs in directed cell differentiations. PPs and NSCs were chosen because of their value in potential applications. In order to generate PPs, iPSCs were firstly treated with a combination of activin A and Wnt3a to initiate the definitive endoderm cell (DE) differentiation, a step resulting in $95 \%$ endodermal progenitors coexpressing FOXA2 and SOX17 (Fig. 1I and 1J). After treating the endodermal progenitors with Retinoic acid, FGF10, Noggin, and Cyclopamine-KAAD, we obtained a highly pure population of PPs positive for the pancreatic progenitor marker PDX1 (Fig. 1I and 1J). As anticipated, these PPs also expressed NKX6.1 and NGN3. An upregulation of insulin gene (INS) expression at transcriptional level was also observed (Fig. 1I and 1J). Additionally, we differentiated iPSCs toward NSCs by treatment with a chemically defined medium comprising of hLIF, SB431542, CHIR99021, Compound E, and dorsomorphin (Liu et al., 2012). These resulting NSCs expressed typical neural progenitor markers SOX2, PAX6, Musashi, and Nestin (Fig. 1K). As a characteristic feature of neural progenitor cells, these cells were able to efficiently differentiate into MAP2 and TUJ1-positive neurons when exposed to a differentiation medium containing ascorbic acid, dbcAMP, BDNF and GDNF for 14 days (Fig. 1K).

In summary, we demonstrated here that integration-free iPSCs can be efficiently and consistently obtained from noninvasively acquired human EMCs and UCs, even in a defective disease context (i.e. type II DM) (Fig. 1L). Given the fact that a similar strategy has been proven to be effective in reprograming diseased somatic cells inherently resistant to the regular cell reprogramming procedure (Liu et al., 2014), we speculate that this methodology could be universally adaptable for various applications. The autologous PP and NSC derivatives generated from emiPSCs and uiPSCs could be further subjected to animal transplantation studies (Sui et al., 2013; Zhang et al., 2009), and even potentially be applied to human regenerative medicine settings. The robustness and non-invasive nature of our system to generate iPSCs from human samples may enormously fuel the application of human iPSCs towards novel cell therapy as well as applications in disease modeling and drug discovery in the near future.

\section{FOOTNOTES}

This work was supported by National Basic Research Program (973 program) (Nos. 2015CB964800, 2014CB910500, and 2014CB964600), the Strategic Priority Research Program of the Chinese Academy of Sciences (No. XDA01020312), National Natural Science Foundation of China (Grant Nos. 81300677, 81330008, 81271266, 31222039, $31201111,81371342,81471414,81401159,81300261,81422017$, and 21331001), Key Research Program of the Chinese Academy of Sciences (No. KJZD-EW-TZ-L05), National Science and Technology Major Project (No. 2011ZX09307-001-08), Beijing Natural Science Foundation (Nos. 7141005 and 5142016), the Thousand Young Talents Program of China, National Laboratory of Biomacromolecules (Nos. 2013kf05, 2013kf11, and 2014kf02), and State Key Laboratory of Drug Research (No. SIMM1302KF-17). JCIB was supported by G. Harold and Leila Y. Mathers Charitable Foundation, and The Leona M. and Harry B. Helmsley Charitable Trust grant (2012-PG-MED002).

Zhichao Ding, Lina Sui, Ruotong Ren, Yanjun Liu, Xiuling Xu, Lina Fu, Ruijun Bai, Tingting Yuan, Ying Hao, Weiqi Zhang, Huize Pan, Wensu Liu, Han Yu, Concepcion Rodriguez Esteban, Xiaobing Yu, Ze Yang, Jian Li, Xiaomin Wang, Juan Carlos Izpisua Belmonte, Guang-Hui Liu, Fei Yi, and Jing Qu declare that they have no conflict of interest.

All institutional and national guidelines for the care and use of laboratory animals were followed. The individuals in this manuscript 
have signed written informed consent for donating EMCs and UCs for somatic cell reprogramming and iPSC study.

Zhichao Ding ${ }^{1}$, Lina Sui ${ }^{1}$, Ruotong Ren ${ }^{1}$, Yanjun Liu ${ }^{2}$, Xiuling $\mathrm{Xu}^{1}$, Lina $\mathrm{Fu}^{1}$, Ruijun Bai ${ }^{1,3}$, Tingting Yuan ${ }^{1}$, Ying $\mathrm{Hao}^{4}$, Weiqi Zhang ${ }^{1}$, Huize Pan ${ }^{1}$, Wensu Liu ${ }^{1}$, Han Yu ${ }^{1}$, Concepcion Rodriguez Esteban ${ }^{7}$, Xiaobing $\mathrm{Yu}^{5}$, Ze Yang ${ }^{5}$, Jian $\mathrm{Li}^{5}$, Xiaomin Wang ${ }^{6}$, Juan Carlos Izpisua Belmonte ${ }^{7}$, Guang-Hui Liu ${ }^{1,6,8 \bowtie}$, Fei $\mathrm{Yi}^{9 凶}$, Jing Qu ${ }^{4 凶}$

${ }^{1}$ National Laboratory of Biomacromolecules, Institute of Biophysics, University of the Chinese Academy of Sciences, Chinese Academy of Sciences, Beijing 100101, China

2 Department of Endocrinology, 306th Hospital of PLA, Beijing 100101, China

${ }^{3}$ Science and Development, Xtem, Foshan 528000, China

${ }^{4}$ State Key Laboratory of Reproductive Biology, Institute of Zoology, Chinese Academy of Sciences, Beijing 100101, China

${ }^{5}$ Beijing Hospital of the Ministry of Health, Beijing 100073, China

${ }^{6}$ Beijing Institute for Brain Disorders, Beijing 100069, China

7 Gene Expression Laboratory, Salk Institute for Biological Studies, 10010 North Torrey Pines Road, La Jolla, CA 92037, USA

${ }^{8}$ Center for Molecular and Translational Medicine, Beijing 100101, China

${ }^{9}$ Department of Molecular and Cellular Physiology, Stanford University School of Medicine, Stanford, CA 94305, USA

$\triangle$ Correspondence: ghliu@ibp.ac.cn (G.-H. Liu), fyi@stanford.edu (F. Yi), qujing@ioz.ac.cn (J. Qu)

\section{OPEN ACCESS}

This article is distributed under the terms of the Creative Commons Attribution License which permits any use, distribution, and reproduction in any medium, provided the original author(s) and the source are credited.

\section{REFERENCES}

Buganim $\mathrm{Y}$, Markoulaki S, van Wietmarschen $\mathrm{N}$, Hoke $\mathrm{H}, \mathrm{Wu} \mathrm{T}$, Ganz K, Akhtar-Zaidi B, He YP, Abraham BJ, Porubsky D et al (2014) The developmental potential of iPSCs is greatly influenced by reprogramming factor selection. Cell stem cell 15:295309

Hou P, Li Y, Zhang X, Liu C, Guan J, Li H, Zhao T, Ye J, Yang W, Liu $\mathrm{K}$ et al (2013) Pluripotent stem cells induced from mouse somatic cells by small-molecule compounds. Science 341:651-654
Lin T, Ambasudhan R, Yuan X, Li W, Hilcove S, Abujarour R, Lin X, Hahm HS, Hao E, Hayek A et al (2009) A chemical platform for improved induction of human iPSCs. Nat methods 6:805-808

Liu GH, Barkho BZ, Ruiz S, Diep D, Qu J, Yang SL, Panopoulos AD, Suzuki K, Kurian L, Walsh C et al (2011) Recapitulation of premature ageing with iPSCs from Hutchinson-Gilford progeria syndrome. Nature 472:221-225

Liu GH, Qu J, Suzuki K, Nivet E, Li M, Montserrat N, Yi F, Xu X, Ruiz S, Zhang $W$ et al (2012) Progressive degeneration of human neural stem cells caused by pathogenic LRRK2. Nature 491:603-607

Liu GH, Suzuki K, Li M, Qu J, Montserrat N, Tarantino C, Gu Y, Yi F, Xu X, Zhang W et al (2014) Modelling Fanconi anemia pathogenesis and therapeutics using integration-free patient-derived iPSCs. Nat Commun 5:4330

Mali P, Chou BK, Yen J, Ye Z, Zou J, Dowey S, Brodsky RA, Ohm JE, Yu W, Baylin SB et al (2010) Butyrate greatly enhances derivation of human induced pluripotent stem cells by promoting epigenetic remodeling and the expression of pluripotency-associated genes. Stem cells 28:713-720

Okita K, Matsumura Y, Sato Y, Okada A, Morizane A, Okamoto S, Hong H, Nakagawa M, Tanabe K, Tezuka K et al (2011) A more efficient method to generate integration-free human iPS cells. Nat methods 8:409-412

Patel M, Yang S (2010) Advances in reprogramming somatic cells to induced pluripotent stem cells. Stem cell rev 6:367-380

Raya A, Rodriguez-Piza I, Guenechea G, Vassena R, Navarro S, Barrero MJ, Consiglio A, Castella M, Rio P, Sleep E et al (2009) Disease-corrected haematopoietic progenitors from Fanconi anaemia induced pluripotent stem cells. Nature 460:53-59

Sanchez Alvarado A, Yamanaka S (2014) Rethinking differentiation: stem cells, regeneration, and plasticity. Cell 157:110-119

Seki T, Yuasa S, Fukuda K (2012) Generation of induced pluripotent stem cells from a small amount of human peripheral blood using a combination of activated T cells and Sendai virus. Nat Protoc 7:718-728

Sui L, Liu GH, Izpisua Belmonte JC (2013) hESC-derived pancreatic progenitors. Cell res 23:592-594

Xue Y, Cai X, Wang L, Liao B, Zhang H, Shan Y, Chen Q, Zhou T, Li $X$, Hou $J$ et al (2013) Generating a non-integrating human induced pluripotent stem cell bank from urine-derived cells. PloS One 8:e70573

Zhang D, Jiang W, Liu M, Sui X, Yin X, Chen S, Shi Y, Deng H (2009) Highly efficient differentiation of human ES cells and iPS cells into mature pancreatic insulin-producing cells. Cell res 19:429-438
Zhichao Ding, Lina Sui, Ruotong Ren, and Yanjun Liu have contributed equally to this work

Electronic supplementary material The online version of this article (doi:10.1007/s13238-014-0117-1) contains supplementary material, which is available to authorized users. 